\title{
Stepping into the ring: the SIN takes on contractile ring assembly
}

\author{
Rachel H. Roberts-Galbraith ${ }^{1,2}$ and Kathleen L. Gould ${ }^{1,2,3}$ \\ ${ }^{1}$ Howard Hughes Medical Institute, Vanderbilt University School of Medicine, Nashville, Tennessee 37232, USA; \\ ${ }^{2}$ Department of Cell and Developmental Biology, Vanderbilt University School of Medicine, Nashville, Tennessee 37232, \\ USA
}

The septation initiation network (SIN) regulates the timing of septum formation in Schizosaccharomyces pombe. However, whether and how the SIN functions in contractile ring formation has remained unclear. In this issue of Genes \& Development, Hachet and Simanis (pp. 3205-3216) demonstrate that the SIN acts downstream from the Plo1 kinase to control a final step in contractile ring assembly. Furthermore, their careful analysis of contractile ring formation may help bridge two existing models of cytokinetic ring formation.

Cell division involves major rearrangements of the cell membrane and cytoskeleton that must be closely coordinated with other cell cycle events. Despite significant advances, several major questions remain regarding how cells build their division machinery and how division is regulated to occur only after DNA has been packaged safely for the two daughter cells.

Schizosaccharomyces pombe provides many advantages as a model organism to address major questions in the cytokinesis field. In addition to being genetically and biochemically tractable, $S$. pombe cells divide symmetrically through the formation and constriction of an actomyosin-based contractile ring (Marks and Hyams 1985; May et al. 1997). Genetic screens allowed identification of many genes whose products are required for cytokinesis (for reviews, see Balasubramanian et al. 2004; Krapp et al. 2004; Wolfe and Gould 2005). These genes encode products that have conserved roles in cytokinesis and/or the actin cytoskeleton in metazoans, including actin, profilin (Cdc3), formin (Cdc12), type II myosin (Myo2) and its light chains (Cde4 and Rlc1), tropomyosin (Cdc8), IQGAP (Rng2), an anillin-like protein (Mid1), and an F-BAR protein (Cdc15). While a thorough biochemical understanding of how these proteins interact with one another is in its infancy, imaging of both live and fixed

[Keywords: S. pombe; cytokinesis; septation initiation network; Mid1p/ anillin; contractile actomyosin ring]

${ }^{3}$ Corresponding author.

E-MAIL kathy.gould@vanderbilt.edu; FAX (615) 343-0723.

Article is online at http://www.genesdev.org/cgi/doi/10.1101/gad.1748908. cells enabled the generation of two main models for ring formation (Kamasaki et al. 2007; Vavylonis et al. 2008), which might be united in light of new information from Hachet and Simanis (2008).

Genetic experimentation also revealed a signaling pathway required for $S$. pombe cytokinesis, the septation initiation network (SIN), which is related to the mitotic exit network (MEN) of Saccharomyces cerevisiae. Although mutation of MEN components results in late mitotic arrest without cytokinesis, SIN mutant cells are unable to divide but still undergo rounds of nuclear division, indicating that mitotic exit and reentry do occur (for a more thorough comparison of the MEN and SIN, see Bardin and Amon 2001; McCollum and Gould 2001). Despite the cell division failure of SIN mutant cells, the precise influence of the SIN on contractile ring formation has remained unclear. Initial recruitment of a variety of contractile ring components to the division site has been observed in SIN mutants, suggesting on the one hand that the SIN is not involved in contractile ring assembly. On the other hand, precocious SIN signaling in interphase is sufficient for contractile ring formation, constriction, and septation, suggesting that the SIN must be involved in some aspect of contractile ring assembly.

Using live cell imaging, Hachet and Simanis (2008) now provide evidence that there is indeed a specific role for the SIN in contractile ring formation that involves recruitment of the essential cytokinetic F-BAR protein, Cdc15, to the medial region of the cell. These observations indicate that the SIN is likely to be active earlier in the cell cycle than previously considered. Furthermore, Hachet and Simanis (2008) provide evidence that Mid1 and the SIN function in parallel downstream from Plol kinase during contractile ring assembly.

Nodes and asters in contractile ring formation

In $S$. pombe, an important step in cell division is the central positioning of the contractile ring such that equivalently sized daughters are formed following abscission. Mid1, a protein possibly related to anillin proteins, is nonessential; however, mid1s cells form mis- 
placed and misoriented rings (Chang et al. 1996; Sohrmann et al. 1996). Mid1 is predominantly nuclear during interphase and localizes to the medial region of the cell cortex upon mitotic entry, cued by the position of the centrally positioned nucleus (Sohrmann et al. 1996; Paoletti and Chang 2000). Mid1 binds the membrane using an amphipathic helix to form a broad, medial band of spots, hereafter referred to as nodes (Chang et al. 1996; Sohrmann et al. 1996; Bahler et al. 1998; Celton-Morizur et al. 2004; Wu et al. 2006). Mid1 then recruits other contractile ring components, possibly through an interaction with type II myosin Myo2 (Motegi et al. 2004), which localizes at nodes at the same time as Mid1, although this interaction may not be direct (Motegi et al. 2000; Wu et al. 2003).

A handful of other proteins essential for cytokinesis localizes to the medial region during the earliest stages of ring formation, including Myo2 and its light chains Rlc1 and Cdc4, IQGAP Rng2, PCH family member Cdc15, and formin Cdc12 (Wu et al. 2003). Several of these proteins colocalize in nodes that coalesce into rings during metaphase and early anaphase $(\mathrm{Wu}$ et al. 2006). Building on this observation and a quantification of contractile ring components (Wu and Pollard 2005), a model was created to describe formation of the contractile ring from nodes (Fig. 1; Vavylonis et al. 2008). The model predicts a "search, capture, pull, and release" mechanism, in which Cdc12 in one node nucleates an actin filament that is captured transiently by Myo2 in a nearby node, allowing nodes to be pulled closer to

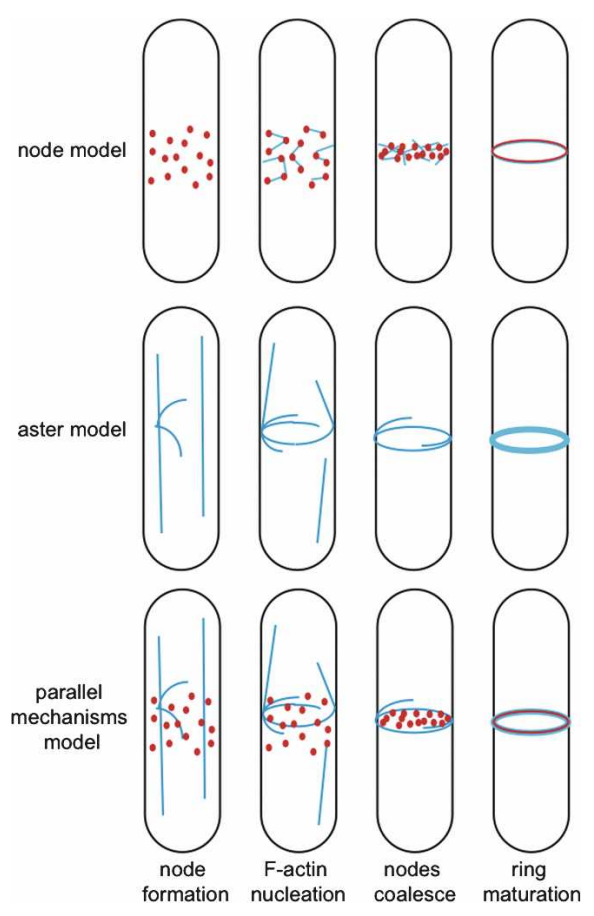

Figure 1. Key elements of the node and aster models are diagrammed. F-actin filaments are shown in blue, and nodes are shown in red. A unified model in which both models contribute in parallel to contractile ring formation is also shown. one another to promote ring formation (Vavylonis et al. 2008).

The only component of the nodes known to be required for their formation is Mid1 (Wu et al. 2006), and the ability of mid1s cells to survive and complete cytokinesis (Chang et al. 1996; Sohrmann et al. 1996) indicates that nodes are not a prerequisite for ring formation or cell division. Despite a slower rate of ring formation in mid1s cells, rings can even become orthogonal given adequate time and increased SIN signaling (Huang et al. 2008). Indeed, precocious SIN signaling during interphase triggers ring formation and cytokinesis in the absence of Mid1 and nodes (Wachtler et al. 2006; Hachet and Simanis 2008). Further, because Mid1 is also necessary for recruitment of Cdc14-like phosphatase Clp1 and Polo-like kinase Plo1 (and possibly other regulators) to the contractile ring (Bahler et al. 1998; Clifford et al. 2008), it is difficult to attribute the mid1s phenotype to node absence alone.

Imaging of F-actin in fixed cells (Arai and Mabuchi 2002) and more recent three-dimensional reconstructions of F-actin filaments in the $S$. pombe cytokinetic ring by electron microscopy (Kamasaki et al. 2007) suggest a different mechanism(s) of contractile ring formation in $S$. pombe. These studies provided evidence that F-actin is initially nucleated as a leading cable or aster with most filaments emanating from one side of the cell (the aster model) (Fig. 1). No meshwork of short F-actin filaments, which would be consistent with the node model of ring assembly, could be seen early in ring formation in these analyses, but instead, long F-actin filaments were observed to encircle the cell early in ring formation, and these filaments coalesced and became reoriented during ring maturation. A consideration here, though, is whether cell fixation influenced the filaments that could be detected in these analyses.

The node and aster models differ primarily in the origin of formin Cdc12-nucleated F-actin in the early ring. Cdc12 localization at one or a few spots would favor the aster model, while the node model requires that Cdc12 be present and active at most nodes. Different groups have reached different conclusions on this point, possibly because the protein is very low abundance and is challenging to detect by live cell imaging. Imaging cells mildly overexpressing $\mathrm{Cdc} 12$ or $\mathrm{Cdc} 15$ has revealed the formation of a single, quite prominent, medially placed Cdc12 spot prior to ring formation (Chang et al. 1997; Carnahan and Gould 2003). Endogenous tagging with three copies of GFP has in one study revealed formation of a few Cdc12-positive nodes after release from a G2 $c d c 25-22$ arrest (Wu et al. 2006), although node formation was not detected in untreated cells in another study (Yonetani et al. 2008). Yet another study attributes the detection of Cdc12 in nodes to the use of latrunculin A (Huang et al. 2008). Thus, this fundamental issue remains to be satisfactorily resolved. Even after it is, the mechanism of Cdc12 activation at spots and/or nodes will also need to be elucidated to support nucleation of actin filaments from a few or many 
loci. Unlike many other formins, there is as yet no evidence that Cdc12 is regulated by autoinhibition that is relieved by small GTPases (Yonetani et al. 2008).

An additional consideration in distinguishing the node and aster models involves Myo2 motor activity. Myo2 clearly localizes to nodes prior to ring formation (Naqvi et al. 1999; Motegi et al. 2000; Wu et al. 2003). However, the node model hinges on Myo2 motor activity driving node convergence, whereas formation of the contractile ring through an aster might not require Myo2 activity initially and might also involve additional forces provided by actin-binding and cross-linking proteins. A key question then is when does Myo2 become active during cell division? Myo2 gliding activity requires the activity of Rng3 in vitro, and Rng3 has not been detected in nodes in wild-type cells (Lord and Pollard 2004; Lord et al. 2008). While these data appear incongruent with the idea that Myo2 drives the capture and convergence of nodes, it remains possible that Rng3 is simply below the level of detection at nodes in wild-type cells. It has also been demonstrated that the motor activity of Myo2 is required for assembly of a continuous ring structure, though ring fragments do appear in the mutant (Naqvi et al. 1999).

\section{SIN signaling and the early contractile ring}

The appearance and organization of these contractile ring components in the middle of the cell need to be tightly regulated to prevent spurious ring formation. One candidate for control of contractile ring formation is the SIN, which is required for cell division in S. pombe. A great deal of work has been done to characterize and order components of the SIN (for reviews, see Balasubramanian et al. 2004; Krapp et al. 2004; Wolfe and Gould 2005). The SIN signal begins with a GTPase, Spg1, which is held in check by a bipartite GAP (GTPase-activating protein) composed of Byr4 and Cdc16. Two scaffolding proteins, Sid4 and Cdc11, tether Spg1 to the spindle pole body (SPB), and Spg1 activation triggers a kinase cascade composed of Cdc7, the Sid1-Cdc14 complex, and the Sid2-Mob1 complex. Temperature-sensitive mutants of positive SIN components result in multinucleate cells due to failure of cell division despite multiple rounds of nuclear division. While this phenotype clearly implicates the SIN in cytokinesis and/or septation in $S$. pombe, many contractile ring components localize medially in SIN mutants, favoring a role for the SIN in contractile ring maintenance or signaling of septum formation.

Using live cell imaging, Hachet and Simanis (2008) have examined the details of $S$. pombe contractile ring formation (visualized by GFP-tagged Rlc1), with specific interest in the impact of the SIN. In agreement with previous studies (Motegi et al. 2004; Wu et al. 2006), they report that ring formation can be observed in distinct stages. First, certain CAR components can be observed as spots or nodes. Then, nodes coalesce into a discontinuous ring and, finally, the ring forms a continuous, "homogeneous," and compact structure during ana- phase. The transition to a more compact ring parallels reorganization of actin during this stage of mitosis (Kamasaki et al. 2007). Hachet and Simanis (2008) report that although ring components still localize medially in SIN mutants, as observed previously, they form a discontinuous ring that fails to become properly organized and quickly disassembles. The amount of at least one component, Cdc15, recruited to these discontinuous structures is also reduced. Furthermore, if the SIN is hyperactivated in interphase, ring assembly proceeds through aster-like structures in the absence of nodes. Interestingly, however, ring contraction under this condition is slower. This might be due to the initial absence of nodes, the absence of Midl itself in these rings, the absence of Midl-interacting proteins such as Plol and Clp1 during ring formation, or the absence of other regulatory influences specific to mitotic cells. When both Mid1 and the SIN are absent in mitotic cells, Hachet and Simanis (2008) find that ring components fail to localize altogether, suggesting that the SIN mediates node-independent contractile ring assembly. Huang et al. (2008) reach a similar conclusion that the SIN is involved in contractile ring organization in the absence of nodes. That two parallel pathways of contractile ring formation function together in $S$. pombe may not be surprising, considering the evolutionary pressure to ensure proper cell division, and it will be interesting to determine whether similar redundancy exists in other eukaryotic organisms.

Hachet and Simanis (2008) show that Cdc15 medial recruitment is severely affected in the absence of Sid2 activity. Given Cdc15's essential function in cytokinesis (Fankhauser et al. 1995), Cdc15 recruitment is likely to be a major effector of SIN function in ring assembly; this model is consistent with the observation that cells lacking Midl and Cdc15 function cannot recruit Rlclp to incipient ring structures (Wachtler et al. 2006). One mechanistic output of SIN signaling is Cdc15 dephosphorylation that is correlated with its medial recruitment during mitosis (Fankhauser et al. 1995; Hachet and Simanis 2008). While it is clear that Cdc15 recruitment is less robust in the absence of SIN signaling, Cdc15 recruitment is unlikely to be the only downstream effector of the SIN important for ring assembly, because although Cdc15 overexpression triggers medial F-actin filament formation in interphase-arrested cells, it is insufficient to trigger proper ring formation.

Therefore, though we now learn that the SIN acts earlier in cytokinesis than frequently considered, the molecular basis for the role of the SIN in contractile ring formation remains unknown. A major deficiency in our understanding is the absence of identified SIN kinase substrates. Based on genetic epitasis and biochemical analysis, Cdc7 is the most upstream kinase in the SIN, the only known effector of activated Spg1. While the activity of Cdc7 remains constant during the cell cycle, both Cdc7 localization to the SPB and Cdc7 interaction with Spg1 occur only in mitosis. Cdc7 is therefore potentially active at both SPBs during metaphase and then at only one SPB during anaphase because Spg1 is inacti- 
vated at one SPB when Cdk1 activity drops. Although suspected targets of Cdc7 include the SIN scaffolding protein Cdc11 and Sid2-Mob1 (based on analogy of the relationship between MEN orthologs), these have not been verified biochemically, and relevant Cdc7 targets are unknown. Sid1, which acts with its cofactor Cdc14, localizes to the SPB and has oscillating kinase activity that peaks during anaphase in a Spg1- and Cdc7-dependent manner. Again, targets of this kinase are unknown. Finally, the Sid2-Mob1 kinase complex exhibits cell cycle-regulated kinase activity that depends on all other positive SIN components. Sid2 localizes to the SPBs and also appears at the contractile ring during late anaphase and the septum during cytokinesis, positioning it to be the SIN kinase capable of acting directly on ring components. However, the only direct substrate of Sid2 identified is Cdc14-like phosphatase Clp1 (Chen et al. 2008). Of note, Sid2 recruitment to the contractile ring occurs in late mitosis, and its activity peaks well after ring formation would have occurred. On the other hand, Sid2 exhibits a lower level of kinase activity during early mitosis, and perhaps bimodal kinase activity of Sid2 or the activity of Cdc7 or Sid1 allows the SIN to play separate roles in contractile ring formation, ring constriction, and septation. Because Sid2 phosphorylates the Clp1 phosphatase and Clp1 localizes to the contractile ring much earlier than Sid2 and dephosphorylates substrates such as Cdc15 at the ring, (Chen et al. 2008; Clifford et al. 2008), Clp1 is likely to be one of the direct outputs of the SIN signal that influences contractile ring formation.

A similar lack of substrate identification limits our understanding of how the MEN impacts cytokinesis in $S$. cerevisiae, and therefore studies from $S$. cerevisiae have not provided clues as to relevant targets in $S$. pombe. Fundamentally, MEN signaling activates and/or directs localization of the phosphatase Cdc14 for mitotic exit, but the MEN also functions in cytokinesis (for review, see Bardin and Amon 2001). As with the SIN, MEN signaling begins with a GTPase, Tem1, which is kept inactive through the action of a bipartite GAP composed of Bub2-Bfa1. A guanine exchange factor (GEF), Lte1, activates Tem 1 on the old SPB as it enters the bud, promoting activation of downstream kinase Cdc15 (not to be confused with the F-BAR protein important for $S$. pombe cytokinesis). Cdc15 kinase phosphorylates and activates Sid2-like kinase Dbf2. Like Sid2, Dbf2 localizes to the SPBs and to the division site in late mitosis. It is not yet known whether the function of the MEN in cytokinesis is Cdc14-dependent, but no contractile ring substrates of MEN kinases or Cdc14 are known.

In light of the missing connection between SIN signaling and specific phosphorylation events leading to changes at the contractile ring, it may be useful to focus on the $S$. pombe ring components known to be phosphoproteins. Mid1 and the F-BAR protein Cdc15 undergo mitotic phosphorylation and mitotic dephosphorylation, respectively (Fankhauser et al. 1995; Sohrmann et al. 1996). As shown in the current work, hyperactivation of the SIN induces hypophosphorylation of Cdc15, possibly through Clp1 (Chen et al. 2008), which dephosphorylates
Cdc15 in vivo and in vitro (Wachtler et al. 2006; Clifford et al. 2008). Mid1 phosphorylation peaks during mitosis, positioning it to be a target of a SIN kinase (Sohrmann et al. 1996), but Midl does not localize in ectopic interphase contractile rings triggered by SIN hyperactivation, arguing that Mid1 is not a key SIN target in ring assembly. Both phosphorylation and dephosphorylation of myosin II heavy chain have been reported to influence Myo2 localization; however, these events have not been linked to SIN activity (Motegi et al. 2000; Mulvihill et al. 2001). Unlike in other eukaryotic cells, myosin regulatory light chain phosphorylation is not required for cytokinesis. Therefore, identifying SIN targets and elucidating the consequence of known phosphorylation events at the ring will be central tasks in advancing our understanding of $S$. pombe cytokinesis.

\section{Polo-like kinases as master regulators of cytokinesis}

In S. pombe, a common regulator of the SIN and Mid1 is polo-like kinase Plo1. Polo-like kinases control many aspects of mitotic progression (for review, see Barr et al. 2004). In S. pombe, Plo1 plays an essential role in mitosis, and mutants in plo1 result in spindle assembly defects, as well as failure of actin ring formation (Ohkura et al. 1995). Overproduction of Plo1 in G2 induces contractile ring formation and septation in a SIN-dependent manner (Tanaka et al. 2001), and SIN hyperactivation cannot induce Plo1 activation or localization to the SPB (Mulvihill et al. 1999), together indicating that Plol acts upstream of the SIN. And, unlike SIN hyperactivation, Plo1 overproduction results in Midl accumulation at rings in most cells (Bahler et al. 1998), suggesting that Plol activation, or activation of Plol and the SIN, enables recruitment of Mid1 to the contractile ring.

The activation of Mid1 by Plol is supported by the observations that Mid1 fails to localize to the contractile ring in the absence of Plol and that mid1s-like, misoriented rings form in cells carrying a temperature-sensitive allele of plo1 (Bahler et al. 1998). Overproduction of Plol also causes a mobility shift in Mid1 protein, suggesting that phosphorylation of Mid1 is Plol-dependent (Bahler et al. 1998). Yeast two-hybrid analysis revealed a direct interaction between Mid1 and Plol (Reynolds and Ohkura 2003), but direct phosphorylation of Mid1 by Plol has not been demonstrated. Defying a simple linear pathway placing Mid1 downstream from Plo1, Plo1 fails to localize to the contractile ring in mid1s cells, indicating a more complex functional relationship (Bahler et al. 1998), and dissecting this relationship would advance our understanding of Plol control of $S$. pombe cytokinesis.

How Plol activates the SIN is also unknown, but it is likely to be analogous to activation of the MEN in $S$. cerevisiae. The Polo-like kinase homolog in S. cerevisiae, Cdc5, is required for mitosis and is specifically important for mitotic exit. Cdc5 activates the GTPase Tem 1 by phosphorylating and inhibiting a component of its GAP, Bfal (for review, see Bardin and Amon 2001). By analogy then, $S$. pombe Plol is likely to activate Spg1 by 
inactivating Byr4-Cdc16, and Byr4 is a known phosphoprotein (Krapp et al. 2008). In addition to its influence on MEN signaling, Cdc5 localizes to the contractile ring, like Plo1, to promote cytokinesis. At the ring, Cdc5 binds several regulators of Rho GTPases through its polo-box domain, including RhoGEF Tus1, which is an important Cdc5 target in cell division (Yoshida et al. 2006). Rhol functions in cytokinesis in S. cerevisiae and binds to formin Bnil, suggesting that Rhol may, like RhoA in mammalian cells, activate actin nucleation important for cytokinetic ring formation.

In $S$. pombe, the role of Rho GTPases in contractile ring assembly or function is poorly understood. Rho GTPases are essential for cell wall synthesis and septation in S. pombe, and therefore it has been difficult to dissect any defects in the early steps of cell division. However, one GEF for the Rho family, Rgf3, localizes to the contractile ring early in mitosis (Wolfe and Gould 2005), suggesting that Rho GTPases in S. pombe may also function in ring assembly. Advancement in our understanding in this area will require identification of Rho GTPase effectors and dissection of Rho GTPase redundancy.

In mammalian cells, as in yeast, Polo-like kinases are master regulators of the cell cycle, and Plk1 inhibitors block cytokinesis in human cells (for review, see Randall et al. 2007). The best-characterized role of Plk1 during cytokinesis in mammalian cells is its requirement for positioning of RhoA (for review, see Piekny et al. 2005). Plk1 specifically promotes recruitment of Ect2, a GEF required for RhoA localization and activity during cytokinesis, though the specific target of Plk1 in this process is not known. RhoA promotes two main facets of contractile ring formation: (1) actin nucleation through relief of formin autoinhibition, and (2) activation of type II myosin through phosphorylation of myosin light chain by Rho kinase (ROCK).

\section{Toward a unified model of contractile ring formation}

With each advance in genetic analysis, biochemical analysis, protein epitope tagging, and live cell imaging, we learned more about the process of cytokinesis using $S$. pombe as a model organism. Though fundamental issues are unresolved, it is tempting to speculate that the node and aster models of contractile ring formation could be combined in light of the current results and those of earlier studies that demonstrated that F-actin and myosin II can be independently recruited into medially placed discontinuous filamentous structures (Naqvi et al. 1999; Motegi et al. 2000). An integrated model similar to that described in the current work would postulate that nodes containing certain contractile ring components would coalesce not by a meshwork of Factin filaments between nodes, but by attaching to Factin filaments nucleated from a smaller number of asters (Fig. 1). As nodes converge into a discontinuous ring through the action of actin-binding proteins (which may or may not include Myo2), nucleation of F-actin from Cdc12 might become more uniform, creating the ran- domized orientation pattern of F-actin seen by electron microscopy and accounting for the increase in homogeneity observed in the current analysis. Additionally, activation of Myo2 motor activity during later stages of ring formation could reorganize filaments to create a more homogenous and stable ring. In the absence of Mid1-organized nodes, F-actin asters form and are slowly organized into functional, yet misoriented, rings (Huang et al. 2008). A node-independent mechanism, as demonstrated by Hachet and Simanis (2008), can be driven by the SIN, but would normally act in parallel with Midl in wild-type cells to conduct the final step in contractile ring formation.

While great strides have been made in our understanding of $S$. pombe cytokinesis, it remains to be determined whether metazoan cells utilize similar mechanisms to achieve cell division. While the activation of the MEN and SIN pathways by Plo1/Cdc5 in yeast is well characterized, the function or even existence of such pathways in metazoans remains uncertain. Less information is available about the functions of MEN/SIN kinase homologs in metazoans (for reviews, see Balasubramanian et al. 2004; Krapp et al. 2004; Wolfe and Gould 2005), although the few described homologs play conserved roles in mitotic exit. An ortholog of the Tem1 and Spg1 GTPases has not been identified, though GAPCenA in higher eukaryotes is homologous to the Bub2 and Cdc16 GAP components. Screens for cytokinesis regulators in Drosophila and mammalian cells did not identify roles for MEN/SIN homologs, but this may be due to redundancy of components. Because the current study suggests a modular control of cytokinesis in S. pombe, in which SIN-dependent and Mid1-dependent mechanisms act in parallel, it may also be that SIN elements in metazoans exist but may act in discrete modules and not as an integrated pathway. Because Polo homologs appear to control cytokinesis in both yeast and metazoans, it may be useful to focus on these kinases as the hub of all downstream cytokinesis regulators, including SIN-like elements, Rho GTPases, and contractile ring components themselves. S. pombe offers the opportunity to dissect in detail the entire cytokinetic pathway downstream from Plo1. In addition to clarifying the regulation of the SIN and Mid1 by Plo1, it will be important to identify the regulator(s) of formin Cdc12, as well as other factors important in formation and remodeling of the contractile ring, which may include ubiquitin ligases like the SCF/Pof6 complex (Hermand et al. 2003), as well as actin cross-linking and depolymerizing factors $(\mathrm{Na}-$ kano et al. 2001; Wu et al. 2001; Ono et al. 2003; Nakano and Mabuchi 2006; Mukhina et al. 2007). The regulation of these factors and their place in contractile ring formation, maintenance, and constriction will be of great interest in future studies.

In conclusion, Hachet and Simanis (2008) have redefined a role of the SIN in contractile ring formation in $S$. pombe. In this work, they demonstrated that Mid1 and the SIN cooperate in ring assembly, and their data, along with previous evidence, indicate that Plol orchestrates both pathways. Importantly, by dissecting the steps in 
this process, the investigators' data support a unified model of contractile ring formation that combines elements of two existing models. The molecular mechanisms that underlie each step in ring formation remain to be elucidated, but will be forthcoming as the mechanistic details of ring component interactions are revealed. Though the specific molecular mechanisms by which Plol and the SIN control the contractile ring are unknown, F-BAR protein Cdc15 is a candidate, and the phosphoregulation of this and other ring components will certainly be a future focus.

\section{References}

Arai, R. and Mabuchi, I. 2002. F-actin ring formation and the role of F-actin cables in the fission yeast Schizosaccharomyces pombe. J. Cell Sci. 115: 887-898.

Bahler, J., Steever, A.B., Wheatley, S., Wang, Y., Pringle, J.R., Gould, K.L., and McCollum, D. 1998. Role of polo kinase and Midlp in determining the site of cell division in fission yeast. J. Cell Biol. 143: 1603-1616.

Balasubramanian, M.K., Bi, E., and Glotzer, M. 2004. Comparative analysis of cytokinesis in budding yeast, fission yeast and animal cells. Curr. Biol. 14: R806-R818. doi: 10.1016/ j.cub.2004.09.022.

Bardin, A.J. and Amon, A. 2001. Men and sin: What's the difference? Nat. Rev. Mol. Cell Biol. 2: 815-826.

Barr, F.A., Sillje, H.H., and Nigg, E.A. 2004. Polo-like kinases and the orchestration of cell division. Nat. Rev. Mol. Cell Biol. 5: 429-440.

Carnahan, R.H. and Gould, K.L. 2003. The PCH family protein, Cdc15p, recruits two F-actin nucleation pathways to coordinate cytokinetic actin ring formation in Schizosaccharomyces pombe. J. Cell Biol. 162: 851-862.

Celton-Morizur, S., Bordes, N., Fraisier, V., Tran, P.T., and Paoletti, A. 2004. C-terminal anchoring of midlp to membranes stabilizes cytokinetic ring position in early mitosis in fission yeast. Mol. Cell. Biol. 24: 10621-10635.

Chang, F., Woollard, A., and Nurse, P. 1996. Isolation and characterization of fission yeast mutants defective in the assembly and placement of the contractile actin ring. J. Cell Sci. 109: 131-142.

Chang, F., Drubin, D., and Nurse, P. 1997. cdc12p, a protein required for cytokinesis in fission yeast, is a component of the cell division ring and interacts with profilin. J. Cell Biol. 137: 169-182.

Chen, C.-T., Feoktistova, A., Chen, J.-S., Shim, Y.-S., Clifford, D.M., Gould, K.L., and McCollum, D. 2008. The SIN kinase Sid2 regulates cytoplasmic retention of the $S$. pombe Cdc14like phosphatase Clp1. Curr. Biol. 18: 1594-1599.

Clifford, D.M., Wolfe, B.A., Roberts-Galbraith, R.H., McDonald, W.H., Yates III, J.R., and Gould, K.L. 2008. The Clp1/ Cdc14 phosphatase contributes to the robustness of cytokinesis by association with anillin-related Mid1. J. Cell Biol. 181: 79-88.

Fankhauser, C., Reymond, A., Cerutti, L., Utzig, S., Hofmann, K., and Simanis, V. 1995. The $S$. pombe cdc15 gene is a key element in the reorganization of F-actin at mitosis. Cell 82: 435-444.

Hachet, O. and Simanis, V. 2008. Midlp/anillin and the septation initiation network orchestrate contractile ring assembly for cytokinesis. Genes \& Dev. (this issue). doi: 10.1101/ gad.1697208.

Hermand, D., Bamps, S., Tafforeau, L., Vandenhaute, J., and
Makela, T.P. 2003. Skp1 and the F-box protein Pof6 are essential for cell separation in fission yeast. J. Biol. Chem. 278: 9671-9677.

Huang, Y., Yan, H., and Balasubramanian, M.K. 2008. Assembly of normal actomyosin rings in the absence of Midlp and cortical nodes in fission yeast. J. Cell Biol. (in press).

Kamasaki, T., Osumi, M., and Mabuchi, I. 2007. Three-dimensional arrangement of $\mathrm{F}$-actin in the contractile ring of fission yeast. J. Cell Biol. 178: 765-771.

Krapp, A., Gulli, M.P., and Simanis, V. 2004. SIN and the art of splitting the fission yeast cell. Curr. Biol. 14: R722-R730. doi: 10.1016/j.cub.2004.08.049.

Krapp, A., Collin, P., Cano Del Rosario, E., and Simanis, V. 2008. Homeostasis between the GTPase Spglp and its GAP in the regulation of cytokinesis in S. pombe. J. Cell Sci. 121: 601-608.

Lord, M. and Pollard, T.D. 2004. UCS protein Rng3p activates actin filament gliding by fission yeast myosin-II. J. Cell Biol. 167: 315-325.

Lord, M., Sladewski, T.E., and Pollard, T.D. 2008. Yeast UCS proteins promote actomyosin interactions and limit myosin turnover in cells. Proc. Natl. Acad. Sci. 105: 8014-8019.

Marks, J. and Hyams, J.S. 1985. Localization of F-actin through the cell division cycle of Schizosaccharomyces pombe. Eur. J. Cell Biol. 39: 27-32.

May, K.M., Watts, F.Z., Jones, N., and Hyams, J.S. 1997. Type II myosin involved in cytokinesis in the fission yeast, Schizosaccharomyces pombe. Cell Motil. Cytoskeleton 38: 385396.

McCollum, D. and Gould, K.L. 2001. Timing is everything: Regulation of mitotic exit and cytokinesis by the MEN and SIN. Trends Cell Biol. 11: 89-95.

Motegi, F., Nakano, K., and Mabuchi, I. 2000. Molecular mechanism of myosin-II assembly at the division site in Schizosaccharomyces pombe. J. Cell Sci. 113: 1813-1825.

Motegi, F., Mishra, M., Balasubramanian, M.K., and Mabuchi, I. 2004. Myosin-II reorganization during mitosis is controlled temporally by its dephosphorylation and spatially by Mid1 in fission yeast. J. Cell Biol. 165: 685-695.

Mukhina, S., Wang, Y.L., and Murata-Hori, M. 2007. $\alpha$-Actinin is required for tightly regulated remodeling of the actin cortical network during cytokinesis. Dev. Cell 13: 554-565.

Mulvihill, D.P., Petersen, J., Ohkura, H., Glover, D.M., and Hagan, I.M. 1999. Plol kinase recruitment to the spindle pole body and its role in cell division in Schizosaccharomyces pombe. Mol. Biol. Cell 10: 2771-2785.

Mulvihill, D.P., Barretto, C., and Hyams, J.S. 2001. Localization of fission yeast type II myosin, Myo2, to the cytokinetic actin ring is regulated by phosphorylation of a C-terminal coiled-coil domain and requires a functional septation initiation network. Mol. Biol. Cell 12: 4044-4053.

Nakano, K. and Mabuchi, I. 2006. Actin-depolymerizing protein Adf1 is required for formation and maintenance of the contractile ring during cytokinesis in fission yeast. Mol. Biol. Cell 17: 1933-1945.

Nakano, K., Satoh, K., Morimatsu, A., Ohnuma, M., and Mabuchi, I. 2001. Interactions among a fimbrin, a capping protein, and an actin-depolymerizing factor in organization of the fission yeast actin cytoskeleton. Mol. Biol. Cell 12: 35153526.

Naqvi, N.I., Eng, K., Gould, K.L., and Balasubramanian, M.K. 1999. Evidence for F-actin-dependent and -independent mechanisms involved in assembly and stability of the medial actomyosin ring in fission yeast. EMBO J. 18: 854-862.

Ohkura, H., Hagan, I.M., and Glover, D.M. 1995. The conserved Schizosaccharomyces pombe kinase plo1, required to form a 
bipolar spindle, the actin ring, and septum, can drive septum formation in G1 and G2 cells. Genes \& Dev. 9: 1059-1073.

Ono, K., Parast, M., Alberico, C., Benian, G.M., and Ono, S. 2003. Specific requirement for two $\mathrm{ADF} /$ cofilin isoforms in distinct actin-dependent processes in Caenorhabditis elegans. J. Cell Sci. 116: 2073-2085.

Paoletti, A. and Chang, F. 2000. Analysis of midlp, a protein required for placement of the cell division site, reveals a link between the nucleus and the cell surface in fission yeast. Mol. Biol. Cell 11: 2757-2773.

Piekny, A., Werner, M., and Glotzer, M. 2005. Cytokinesis: Welcome to the Rho zone. Trends Cell Biol. 15: 651-658.

Randall, C.L., Burkard, M.E., and Jallepalli, P.V. 2007. Polo kinase and cytokinesis initiation in mammalian cells: Harnessing the awesome power of chemical genetics. Cell Cycle 6: $1713-1717$.

Reynolds, N. and Ohkura, H. 2003. Polo boxes form a single functional domain that mediates interactions with multiple proteins in fission yeast polo kinase. J. Cell Sci. 116: $1377-$ 1387.

Sohrmann, M., Fankhauser, C., Brodbeck, C., and Simanis, V. 1996. The dmf $1 / \mathrm{mid} 1$ gene is essential for correct positioning of the division septum in fission yeast. Genes \& Dev. 10: 2707-2719.

Tanaka, K., Petersen, J., MacIver, F., Mulvihill, D.P., Glover, D.M., and Hagan, I.M. 2001. The role of Plol kinase in mitotic commitment and septation in Schizosaccharomyces pombe. EMBO J. 20: 1259-1270.

Vavylonis, D., Wu, J.Q., Hao, S., O'Shaughnessy, B., and Pollard, T.D. 2008. Assembly mechanism of the contractile ring for cytokinesis by fission yeast. Science 319: 97-100.

Wachtler, V., Huang, Y., Karagiannis, J., and Balasubramanian, M.K. 2006. Cell cycle-dependent roles for the FCH-domain protein Cdc15p in formation of the actomyosin ring in Schizosaccharomyces pombe. Mol. Biol. Cell 17: 3254-3266.

Wolfe, B.A. and Gould, K.L. 2005. Split decisions: Coordinating cytokinesis in yeast. Trends Cell Biol. 15: 10-18.

Wu, J.Q. and Pollard, T.D. 2005. Counting cytokinesis proteins globally and locally in fission yeast. Science 310: 310-314.

Wu, J.Q., Bahler, J., and Pringle, J.R. 2001. Roles of a fimbrin and an $\alpha$-actinin-like protein in fission yeast cell polarization and cytokinesis. Mol. Biol. Cell 12: 1061-1077.

Wu, J.Q., Kuhn, J.R., Kovar, D.R., and Pollard, T.D. 2003. Spatial and temporal pathway for assembly and constriction of the contractile ring in fission yeast cytokinesis. Dev. Cell 5: 723-734.

Wu, J.Q., Sirotkin, V., Kovar, D.R., Lord, M., Beltzner, C.C., Kuhn, J.R., and Pollard, T.D. 2006. Assembly of the cytokinetic contractile ring from a broad band of nodes in fission yeast. J. Cell Biol. 174: 391-402.

Yonetani, A., Lustig, R.J., Moseley, J.B., Takeda, T., Goode, B.L., and Chang, F. 2008. Regulation and targeting of the fission yeast formin cdc12p in cytokinesis. Mol. Biol. Cell 19: 22082219.

Yoshida, S., Kono, K., Lowery, D.M., Bartolini, S., Yaffe, M.B., Ohya, Y., and Pellman, D. 2006. Polo-like kinase Cdc5 controls the local activation of Rhol to promote cytokinesis. Science 313: 108-111. 


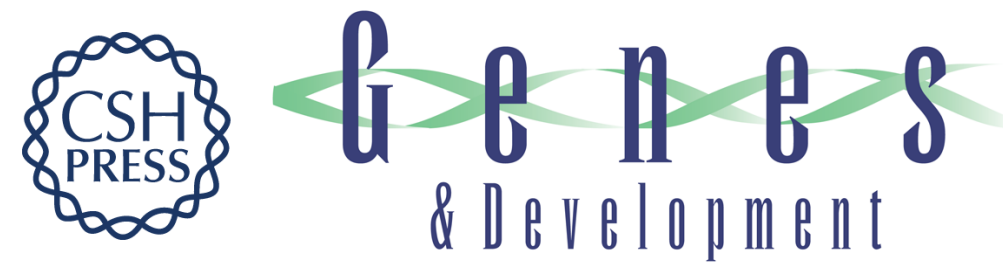

\section{Stepping into the ring: the SIN takes on contractile ring assembly}

Rachel H. Roberts-Galbraith and Kathleen L. Gould

Genes Dev. 2008, 22:

Access the most recent version at doi:10.1101/gad.1748908

\section{Related Content Mid1p/anillin and the septation initiation network orchestrate contractile ring assembly for cytokinesis \\ Olivier Hachet and Viesturs Simanis \\ Genes Dev. November, 2008 22: 3205-3216}

References This article cites 47 articles, 33 of which can be accessed free at:

http://genesdev.cshlp.org/content/22/22/3082.full.html\#ref-list-1

Articles cited in:

http://genesdev.cshlp.org/content/22/22/3082.full.html\#related-urls

\section{License}

Email Alerting Receive free email alerts when new articles cite this article - sign up in the box at the top Service right corner of the article or click here.

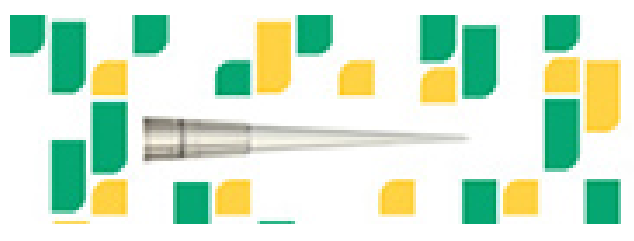

Focused on your science. 